\title{
Cloning and Characterization of the Urea Transporter UT3 Localization in Rat Kidney and Testis
}

\author{
Hiroyasu Tsukaguchi, ${ }^{\star}$ Chairat Shayakul, ${ }^{\star}$ Urs V. Berger, ${ }^{\star}$ Taro Tokui,, Dennis Brown, ${ }^{\ddagger}$ and Matthias A. Hediger* \\ $*$ Renal Division, Brigham and Women’s Hospital, Harvard Medical School, Boston, Massachusetts 02115; and ${ }^{\ddagger}$ Renal Unit, \\ Massachusetts General Hospital, Harvard Medical School, Boston, Massachusetts 02129
}

\begin{abstract}
Urea transport in the kidney plays an important role in urinary concentration and nitrogen balance. Recently, three types of urea transporters have been cloned, UT1 and UT2 from rat and rabbit kidney and HUT11 from human bone marrow. To elucidate the physiological role of the latter urea transporter, we have isolated the rat homologue (UT3) of HUT11 and studied its distribution of expression and functional characteristics. UT3 cDNA encodes a 384 amino acid residue protein, which has $80 \%$ identity to the human HUT11 and $62 \%$ identity to rat UT2. Functional expression in Xenopus oocytes induced a large ( $\sim 50$-fold) increase in the uptake of urea compared with water-injected oocytes. The uptake was inhibited by phloretin $(0.75 \mathrm{mM})$ and pCMBS (0.5 $\mathrm{mM}$ ) (55 and $32 \%$ inhibition, respectively). Northern analysis gave a single band of $3.8 \mathrm{~kb}$ in kidney inner and outer medulla, testis, brain, bone marrow, spleen, thymus, and lung. In situ hybridization of rat kidney revealed that UT3 mRNA is expressed in the inner stripe of the outer medulla, inner medulla, the papillary surface epithelium, and the transitional urinary epithelium of urinary tracts. Costaining experiments using antibody against von Willebrand factor showed that UT3 mRNA in the inner stripe of the outer medulla is expressed in descending vasa recta. These data suggest that UT3 in kidney is involved in counter current exchange between ascending and descending vasa recta, to enhance the cortico-papillary osmolality gradient. In situ hybridization of testis revealed that UT3 is located in Sertoli cells of seminiferous tubules. The signal was only detected in Sertoli cells associated with the early stages of spermatocyte development, suggesting that urea may play a role in spermatogenesis. (J. Clin. Invest. 1997. 99:15061515.) Key words: urea transport • urinary concentration • kidney $\bullet$ testis $\bullet$ spermatocyte
\end{abstract}

\section{Introduction}

Urea is the major end product of nitrogen metabolism in mammals and its transport in kidney plays an important role in urinary concentration (1-3). Urea transport in the kidney inner

Address correspondence to Matthias A. Hediger, Renal Division, Brigham and Women's Hospital and Harvard Medical School, 75 Francis Street, Boston, MA 02115. Phone: 617-732-5850; FAX: 617732-6392; E-mail: mhediger@bustoff.bwh.harvard.edu

Received for publication 16 October 1996 and accepted in revised form 10 January 1997.

J. Clin. Invest.

(C) The American Society for Clinical Investigation, Inc. 0021-9738/97/04/1506/10 \$2.00

Volume 99, Number 7, April 1997, 1506-1515 medulla and in red blood cells is a facilitated process $(4,5)$. $\mathrm{Re}$ cently, we have cloned the urea transporter UT2 from rabbit kidney medulla with functional expression in Xenopus oocytes (6). Subsequent studies of the corresponding urea transporter from rat kidney demonstrated that the rat UT gene encodes two urea transporter splice variants, the originally reported UT2 (2.9 kb mRNA) and UT1 (4.0 kb mRNA) (7-9). UT1 consists of two internal homologous halves, the $\mathrm{NH}_{2}$-terminal half is homologous to rat UT2 (59\% identity) and the COOHterminal half is identical to UT2, indicating that the UT gene evolved from an ancestral gene by gene duplication (9). Our studies indicated that UT1 and UT2 are exclusively expressed in kidney. Interestingly, expression of these isoforms is regulated differently under different physiological conditions. The UT2 and UT1 mRNAs are upregulated by dehydration and protein restriction, respectively $(7,8)$.

Another urea transporter that has $63 \%$ amino acid identity with UT2 was isolated from human bone marrow (HUT11) (10). HUT11 has been shown to correspond to the erythrocyte urea transporter and to represent the Kidd (Jk) blood group antigen (11). A recent study using hydronephrotic kidneys suggested that HUT11 is expressed in vascular structures (12). However, the precise localization of HUT11 in the kidney has not been elucidated.

During antidiuresis, urea is reabsorbed from inner medullary collecting ducts (IMCD), a process regulated by vasopressin (13). Accumulation of urea in the inner medulla is crucial for the kidney to maximize urinary concentration. To establish and maintain the cortico-papillary osmolarity gradient, urea absorbed in the inner medullary collecting ducts is recycled by secretion into thin descending limbs (14). Counter current exchange of urea between descending and ascending vasa recta (AVR and DVR $)^{1}$ and between descending limbs of Henle loops and AVR is furthermore thought to limit the escape of urea from the inner medulla. Recent studies indicated that there is a phloretin-inhibitable urea transporter in the DVR (15). AVR do not need a urea transporter, because their endothelial cells are fenestrated (16).

Recent localization studies using reverse transcriptase PCR (17) from isolated nephron segments and immunocytochemistry (18) demonstrated that UT2 is expressed in thin descending limbs of short loops of Henle, suggesting that UT2 provides a pathway of urea reentry into the descending limbs. The urea permeabilities are different in tubule segments and blood vessels $(4,19)$. Likewise, different types of urea transporters may participate in urea recycling and counter current exchange by analogy with the aquaporin family of water channels, which includes several renal isoforms involved in urinary concentration $(20,21)$.

1. Abbreviations used in this paper: AVR, ascending vasa recta; DVR, descending vasa recta. 
The aim of this study was to characterize the tissue distribution and physiologic role of the erthrocyte urea transporter by studying its rat homologue and to test whether there are additional urea transporter isoforms expressed in rat kidney. A PCR-based homology cloning approach was used and degenerative primers were designed corresponding to conserved regions of the UT family. This led to the isolation of the kidney urea transporter UT3, which appears to be the rat homologue of HUT11. UT3 is strongly expressed in the kidney, consistent with a recent finding based on a partial clone (12). UT3 is furthermore expressed in testis, brain, bone marrow, and spleen. In the present study, we have undertaken a detailed analysis of the expression of UT3 in the kidney. We also report its expression in the rat testis and we propose a potential role of urea transporters in spermatogenesis.

\section{Methods}

PCR amplification and cDNA library screening. Rat kidney poly $\left(\mathrm{A}^{+}\right)$ RNA $(1 \mu \mathrm{g})$ was reverse transcribed using oligo dT primer and Superscript II RNase H-reverse transcriptase (GIBCO BRL, Gaithersburg, MD). The following degenerative primer pair which corresponds to to a highly conserved hydrophobic region in UT2 and HUT11 was designed $(7,10)$ : sense primer, $5^{\prime}-\mathrm{GT}(\mathrm{G} / \mathrm{A} / \mathrm{T} / \mathrm{C}) \mathrm{CA}(\mathrm{A} /$ G)AA(T/C)CC(G/A/T/C)TGGTGGGC-3' (nucleotides 1184-1203, in rat UT2), antisense primer, $5^{\prime}-\mathrm{A}(\mathrm{A} / \mathrm{G})(\mathrm{A} / \mathrm{G}) \mathrm{CA}(\mathrm{A} / \mathrm{G}) \mathrm{AA}(\mathrm{G} / \mathrm{A} / \mathrm{T} /$ C)GGCCA(G/A/T/C)GT(A/G)CA-3' (nucleotides 1936-1955, in rat UT2). Reverse-transcribed RNA was amplified in a $20 \mu \mathrm{l}$ reaction with 100 pmol of primers. The reaction was carried out using a thermal DNA cycler (4800; Perkin Elmer Corp., Norwalk, CT) for 35 cycles. Each cycle comprised denaturation at $95^{\circ} \mathrm{C}$ for $10 \mathrm{~s}$, annealing at $55^{\circ} \mathrm{C}$ for $60 \mathrm{~s}$, and extension at $72^{\circ} \mathrm{C}$ for $60 \mathrm{~s}$. The PCR products of the expected size $(750 \mathrm{bp})$ were gel purified and subcloned into the pCRII vector (Invitrogen, San Diego, CA). The inserted PCR products were sequenced by the dideoxy-termination method using Sequenase 2.0 sequencing system (USB Biologicals, Cleveland, $\mathrm{OH}$ ). One of the clones (UT3/\#10) had a significant homology to the reported urea transporters UT2 and HUT11 and was used as a probe for screening of a rat inner medulla cDNA library prepared using the Superscript Cloning System (GIBCO BRL). For library screening, the EcoRI fragment of the UT3/\#10 clone was labeled with ${ }^{32} \mathrm{P}-\mathrm{dCTP}$, using the T7 Quick Prime kit (Pharmacia LKB Biotechnology Inc., Piscataway, NJ). Screening was performed under high stringency conditions that included washing with $0.1 \times \mathrm{SSC}, 0.1 \% \mathrm{SDS}$, at $65^{\circ} \mathrm{C}$. A positive clone was selected and subcloned into pBluescript II SK(-) (Stratagene, La Jolla, CA).

Northern analysis. Total RNA was prepared from male SpragueDawley rats by the guanidinium isothianate method using cesium trifluoroacetic acid (Pharmacia LKB Biotechnology Inc.). Poly $\left(\mathrm{A}^{+}\right)$ RNA was purified by oligo-dT cellulose chromatography. Total RNA $(10 \mu \mathrm{g})$ or Poly $\left(\mathrm{A}^{+}\right)$RNA $(3 \mu \mathrm{g})$ were electrophoresed on a $1 \%$ formaldehyde agarose gel and blotted onto a nylon filter. The full length UT3 cDNA was excised from pBluescript II using the restriction enzyme NotI and labeled with ${ }^{32} \mathrm{P}-\mathrm{dCTP}$. The filter was hybridized at $42^{\circ} \mathrm{C}$ for $18 \mathrm{~h}$ in $50 \%$ formamide hybridization and washed at high stringency $\left(0.1 \times \mathrm{SSC} / 0.1 \% \mathrm{SDS}\right.$ at $\left.65^{\circ} \mathrm{C}\right)$.

In vitro transcription and functional characterization. Sense UT3 cRNA was synthesized from UT3 cDNA in pBluescript SK (-) after linearization with SacII, using T7 RNA polymerase. Rat UT2 and HUT11 cRNA were prepared as previously described $(7,10)$. Urea uptake studies in Xenopus oocytes were performed as previously reported $(6,7)$. In brief, $\sim 50 \mathrm{ng}$ of UT3 cRNA was injected into collagenase-treated and manually defolliculated oocytes. After incubation at $18^{\circ} \mathrm{C}$ in Barth medium for $2-3 \mathrm{~d}$, oocytes were preincubated at room temperature for $1 \mathrm{~h}$ in uptake solution (200 mM Mannitol, 2 $\mathrm{mM} \mathrm{KCl}, 1 \mathrm{mM} \mathrm{MgSO}_{4}, 1 \mathrm{mM} \mathrm{CaCl} 2,10 \mathrm{mM}$ Hepes, $5 \mathrm{mM}$ Tris $\mathrm{pH}$
7.4). To measure urea uptake, oocytes were incubated for $90 \mathrm{~s}$ with uptake solution including $2.7 \mu \mathrm{Ci}\left[{ }^{14} \mathrm{C}\right]$ urea/ml and $1 \mathrm{mM}$ urea. The uptake was terminated by adding ice cold uptake solution containing $1 \mathrm{mM}$ unlabeled urea. Oocytes were washed five times with the same solutions and dissolved in $200 \mu \mathrm{l}$ of $10 \%$ SDS. Radioactivity of the lysate was counted using a scintillation counter. For inhibition studies with urea analogues, oocytes were preincubated for $30 \mathrm{~min}$ in uptake solution containing $50 \mathrm{mM}$ mannitol and $150 \mathrm{mM}$ of urea analogues (thiourea, 1,1-dimethyl thiourea, or 1,3-dimethyl thiourea; Sigma Chemical Co., St Louis, MO), and uptake of urea (1 mM) was measured during $90 \mathrm{~s}$. For inhibition studies with phloretin or para-chloromercuribenzene sulfonate (pCMBS; Sigma Chemical Co.), uptake of urea $(1 \mathrm{mM})$ was measured in the presence of $200 \mathrm{mM}$ mannitol and either $0.7 \mathrm{mM}$ phloretin or $1 \mathrm{mM}$ pCMBS.

In situ hybridization. Digoxigenin-labeled antisense and sense cRNAs were synthesized from linearized plasmids containing the complete UT3 cDNA using the Genius Kit (Boehringer-Mannheim, Indianapolis, IN) according to the manufacturer's instructions. Transcripts were alkalihydrolyzed to an average length of 300-600 base. In situ hybridization was performed on cryosections $(5-10 \mu \mathrm{m})$ of freshly frozen kidneys and testes, based on the protocol described by Schaeren-Wiemers and Gerfin-Moser (22). The hybridization buffer consisted of $50 \%$ formamide, $5 \times$ SSC, $2 \%$ blocking reagent (Boehringer-Mannheim), $0.02 \%$ SDS, and $0.1 \% N$-laurylsarcosine, and probe $(\sim 50 \mathrm{ng} / \mathrm{ml})$. Sections were cover-slipped and hybridization was allowed to proceed at $68^{\circ} \mathrm{C}$ for $18 \mathrm{~h}$. After hybridization, sections were washed three times for $5 \mathrm{~min}$ in $2 \times \mathrm{SSC}$ and twice for $30 \mathrm{~min}$ in $0.1 \times \mathrm{SSC}$ at $68^{\circ} \mathrm{C}$. The hybridized digoxigenin-labeled probes were visualized using antidigoxigenin Fab fragments (Boehringer-Mannheim) and BCIP/NBT substrate as described (22). The sections were developed for $18 \mathrm{~h}$ before they were either mounted in glycerol-gelatin PBS or processed for double staining.

To localize the UT3 signal to vascular cells in the kidney, 5- $\mu \mathrm{m}$ adjacent sections were double stained with an antiserum against von Willebrand Factor VIII (23) (prediluted solution; Zymed, South San Francisco, CA). The von Willebrand antibodies were detected with horseradish peroxidase-conjugated anti-rabbit secondary antibodies (20 $\mu \mathrm{g} / \mathrm{ml}$; Jackson ImmunoResearch Labs, Inc., West Grove, PA) followed by a standard $\mathrm{DAB}$ reaction. To determine the maturation state of the spermatocytes in UT3-positive seminiferous tubules of testis, sections were double-labeled with mouse anti- $\alpha$-tubulin antibodies (24) $(20 \mu \mathrm{g} / \mathrm{ml}$; Sigma Chemical Co.), which were detected by a subsequent 1 -h incubation with biotinylated anti-mouse secondary antibodies $(5 \mu \mathrm{g} / \mathrm{ml}$, Jackson Immunoresearch Labs., Inc.) and Cy3streptavidin (1\%, Zymed). Double-stained sections were coverslipped using Vectashield (Vector Lab., Burlingame, CA), and photographed using fluorescence microscopy.

\section{Results}

Molecular cloning of a cDNA from rat kidney inner medulla. PCR amplification using a degenerative primer pair (see Methods) yielded 750-bp PCR products, which were subcloned into the pCRII vector. 20 clones were selected and analyzed by sequencing. One of them (UT3/\#10) showed $71 \%$ amino acid identity to rat UT2 (7). The other clones either represented UT2 or sequences unrelated to UT2. UT3\#10 clone was used as a probe to screen a rat kidney inner medulla $\lambda \mathrm{gt} 10 \mathrm{cDNA}$ library, and the clone with the longest insert was sequenced. It consists of $3.8-\mathrm{kb}$ nucleotides and contains an open reading frame of 1155 nucleotides. The start codon corresponds to a consensus Kozak consensus sequence (25) (CGCCATGG, nucleotides 90-97). The open reading frame is flanked by a 93 bp-long $5^{\prime}$ and a $2.5 \mathrm{~kb}$-long $3^{\prime}$ untranslated region. The cDNA has a poly (A) tail and a polyadenylation site (AATAAA). The UT3 coding sequence predicts a 384 amino acid residue 
A

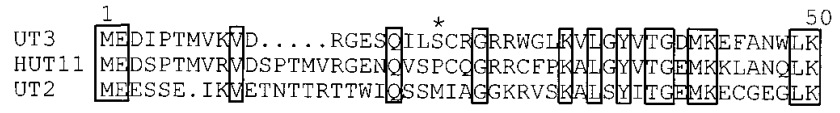

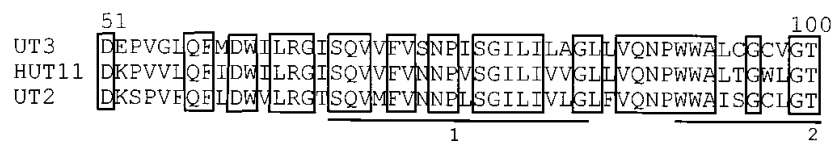

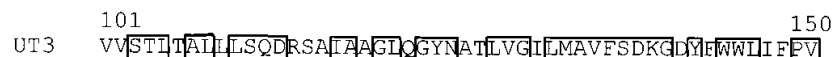
HUT11 VVSTIMAL LSQDRSLIASGLYGYMATL VGULMAVFSDKGDY EWWL L PV

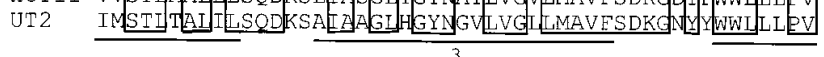
151

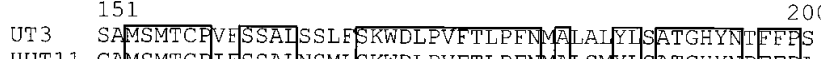

HUT11 CAMSMTCA I SSAT NSML SKWDLPVFTLPFN WX LSM YI SATGHYN PEPA

UT2 IVMSMTCAILSSAISTVESKDLPVFTLPFNIENTLYUNTGHYNEEAT

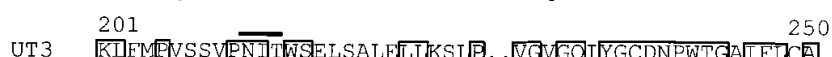

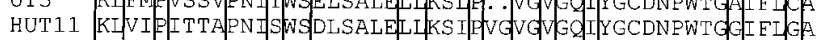

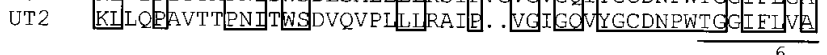

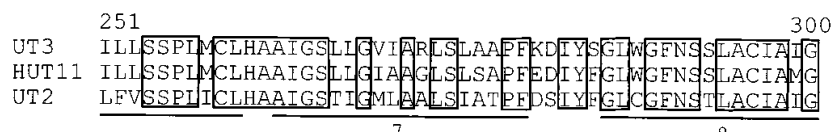

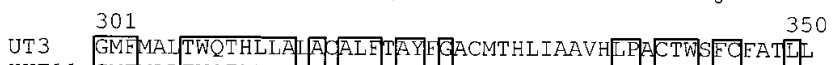

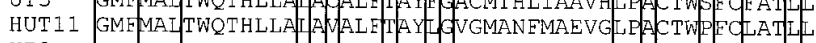

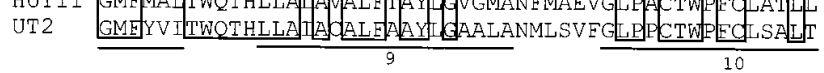

351 10 400

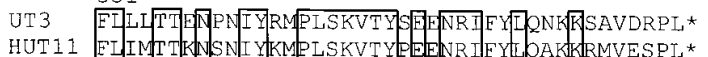

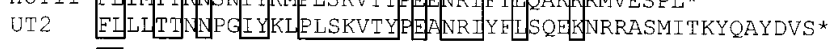

\section{C}

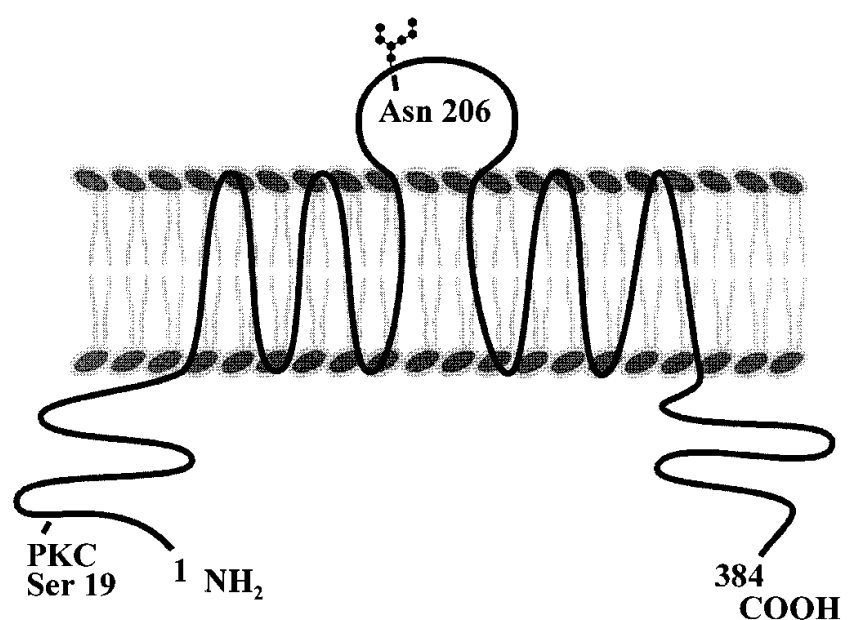

B

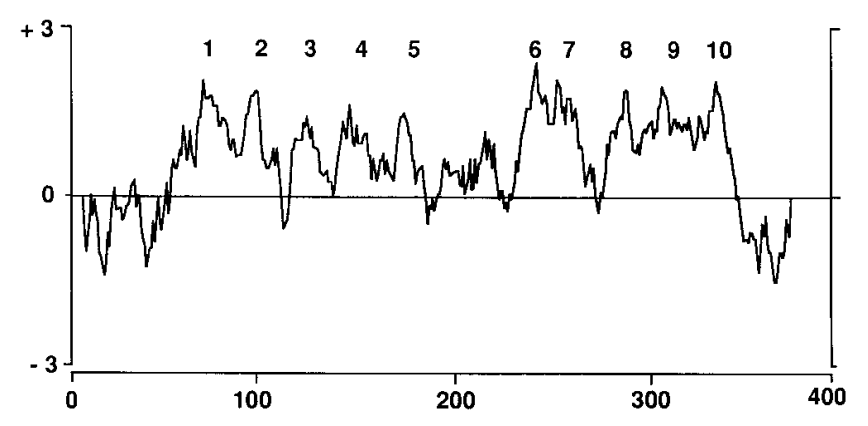

Figure 1. Amino acid sequence of UT3. (A) Deduced amino acid sequence of UT3 and alignments with HUT11 and UT2 $(7,10)$. The bar shows a potential $\mathrm{NH}_{2}$-linked glycosylation site (Asn 206) and the asterisk represents a potential protein kinase $\mathrm{C}$ phosphorylation site (Ser 19). Putative membrane-spanning regions are underlined and numbered 1-10. The sequence has been deposited in the GenBank (accession No. U81518). A partial sequence of UT3 from amino acid 183 to 296 was reported under the name of rUT11 (12). Compared with our cDNA sequence, rUT11 has two amino acid differences at the position of 199 (Met in UT3 vs. Asn in rUT11) and of 266 (Arg in UT3 vs. Gly in rUT11). This could reflect either a misincorporation of nucleotides during PCR-amplification of clone rUT11 or an amino acid polymorphism of UT3/rUT11. (B) Kyte-Doolittle hydropathy analysis of the deduced amino acid sequence of UT3 using a window of 21 amino acids (6). The numbering of putative membrane-spanning regions corresponds to those of $A$. (C) Schematic representation of a hypothetical topology of UT3. Potential sites for $\mathrm{NH}_{2}$-linked glycosylation and $\mathrm{PKC}$ phosphorylation are depicted.

Tissue distribution of UT3. High stringency Northern anal-

peptide (Fig. $1 A$ ) with a predicted molecular mass of $41 \mathrm{kD}$. By analogy with UT2, hydropathy analysis using the KyteDoolittle algorithm suggests the presence of two large hydrophobic domains containing 10 putative transmembrane domains $(6,7)$ (Fig. 1, $B$ and $C$ ). UT3 cDNA has a potential $N$-linked glycosylation site at Asn 206 and a potential protein kinase C (PKC) phosphorylation site at Ser 19, but unlike UT2, there are no protein kinase A (PKA) consensus sites. ysis revealed the presence of a single $3.8 \mathrm{~kb}$ transcript in all positive tissues, suggesting the absence of splice variants (Fig. 2). UT3 mRNA is strongly expressed in kidney inner medulla, outer medulla, and cortex. In contrast to UT1 and UT2 which were found to be kidney specific, UT3 mRNA has a much broader tissue distribution. Signals were detected in testis, thymus, bone marrow, spleen, and to a lesser degree in the lung. 


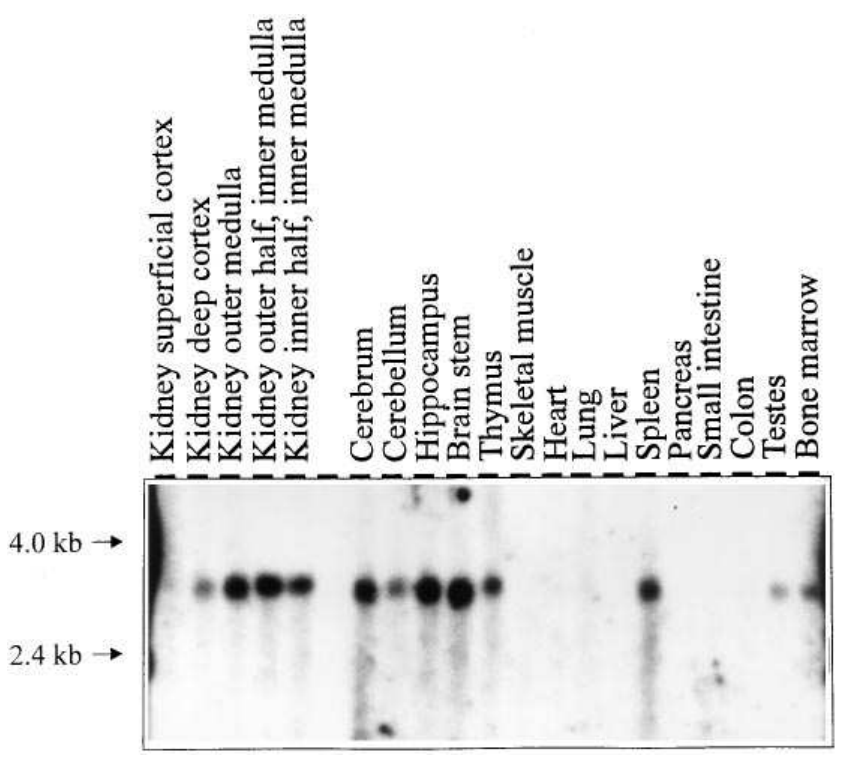

Figure 2. Northern blot analysis of UT3 mRNA from various rat tissues. Total RNA $(10 \mu \mathrm{g})$ from the outer and inner medulla and bone marrow, or poly $\left(\mathrm{A}^{+}\right) \mathrm{RNA}(3 \mu \mathrm{g})$ from the other rat tissues were electrophoresed in a formaldehyde agarose gel and transferred to a nylon membrane. The filter was probed with ${ }^{32} \mathrm{P}$-labeled full length UT3 cDNA and hybridized at $42^{\circ} \mathrm{C}$ and washed with $0.1 \%$ SDS, $0.1 \times \mathrm{SSC}$, at $65^{\circ} \mathrm{C}$. Autoradiograghy was obtained after 5 -d exposure at $-80^{\circ} \mathrm{C}$.

Interestingly, high levels of expression were also found in all regions of the brain including cerebrum, cerebellum, brain stem, and hippocampus. No hybridization signals were detected in liver, colon, heart, and skeletal muscle. Overall, this tissue distribution is consistent with Northern analysis based on a partial cDNA probe (12).

Functional characterization of UT3. The transport characteristics of UT3 were determined in UT3 cRNA injected $\mathrm{Xe}$ nopus oocytes. UT3 expression increased the uptake of urea (1 $\mathrm{mM}) \sim 50$-fold above water-injected control oocytes, resulting in a urea permeability of $\sim 2.5 \times 10^{-5} \mathrm{~cm} / \mathrm{s}$. This value is similar to those determined for UT2 and HUT11 expressed in oocytes $(7,10)$. UT3-mediated urea transport was inhibited 57 , 21 , and $86 \%$, respectively, by the urea analogues, thiourea, 1,1dimethyl-thiourea (DMT) and 1,3-DMT, (150 mM inhibitor concentration) (Fig. $3 A$ ). Inhibition by thiourea was weaker for UT3 than for UT2 and HUT11, which were inhibited by $62 \%$ (150 mM thiourea) and 88\% (50 mM thiourea), respectively, $(7,10)$. Urea uptake by UT3 was blocked $55 \%$ in the presence of $0.7 \mathrm{mM}$ phloretin, a concentration that gave complete inhibition for UT2 and HUT11 (Fig. 3, $B$ and $C$ ). Urea uptake by UT3 was inhibited $32 \%$ in the presence of $0.5 \mathrm{mM}$ pCMBS, whereas HUT11 was inhibited $61 \%$ in the same pCMBS concentration (Fig. 3, $B$ and $C$ ). UT2 showed no sensitivity to $0.5 \mathrm{mM}$ pCMBS (Fig. $3 C$ ). Thus, the inhibition studies indicate a lower sensitivity of UT3 toward phloretin and pCMBS compared with UT2 and HUT11.

Localization of UT3 $m R N A$ in rat kidney. Fig. $4 A$ shows a low-magnification view of longitudinal kidney sections hybridized with UT3 antisense probe. Dense hybridization signals are found in the inner stripe of the outer medulla, inner medulla, and papillary tip. The distribution of UT3 mRNA is con-
A
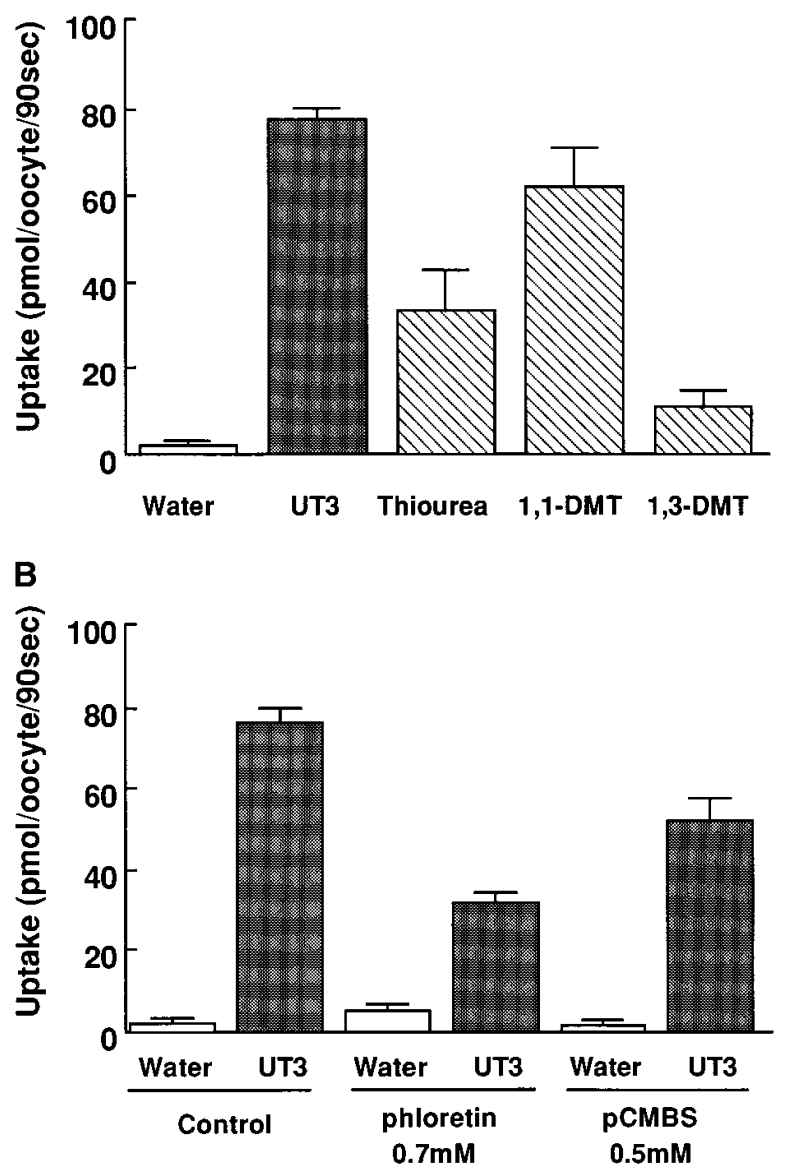

C

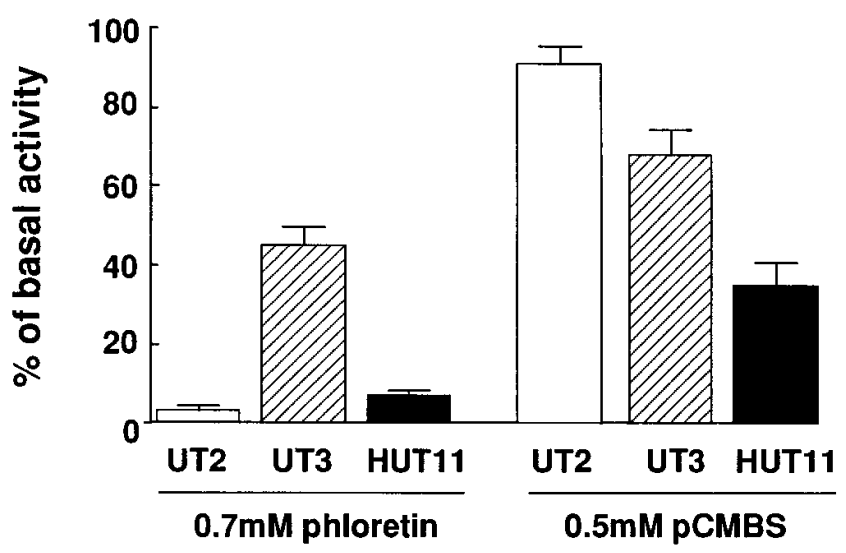

Figure 3. Urea transport of UT3 expressed in Xenopus oocytes. Uptake of urea $(1 \mathrm{mM})$ for $90 \mathrm{~s}$ was measured in Xenopus oocytes injected with water and UT3 cRNA. ( $A$ ) Effects of urea analogues on urea uptake. The urea uptake was measured in the presence of thiourea, 1,1-dimethyl thiourea $(D M T)$, or 1,3-dimethyl thiourea $(D M T)$ (150 mM concentration). (B) Effects of transport inhibitors on urea uptake. The urea uptake was measured in the presence of $0.7 \mathrm{mM}$ phloretin and $0.5 \mathrm{mM}$ pCMBS. $(C)$ Comparison of the effects of transport inhibitors on urea uptake of UT2, UT3, and HUT11. A percentage of urea uptake by UT2, UT3, and HUT11 in the presence of $0.7 \mathrm{mM}$ phloretin or $0.5 \mathrm{mM}$ pCMBS, which is compared with basal uptake in the absence of the inhibitors, is indicated. The bars represent the mean \pm SEM from 6 to 8 oocytes. 

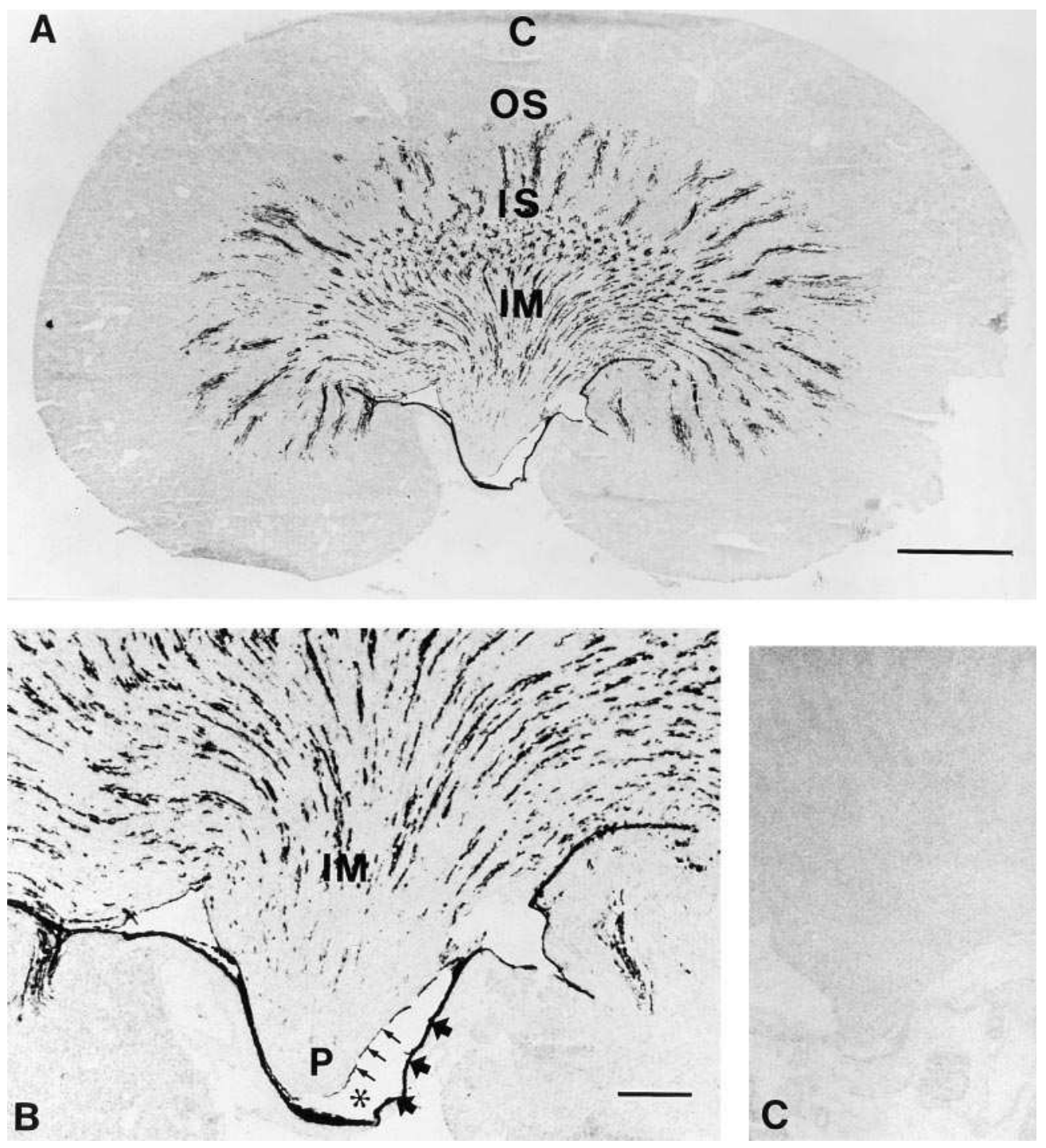

Figure 4. In situ hybridization of UT3 mRNA on rat kidney. (A) Antisense UT3 cRNA probe was hybridized on a longitudinal section of rat kidney. $C$, cortex; $O S$, outer stripe of the outer medulla; $I S$, inner stripe of the outer medulla; $I M$, inner medulla. $\mathrm{Bar}=2 \mathrm{~mm}$. $(B)$ Higher magnification of the inner medulla and the urinary space (asterisk). The papillary surface epithelium (small arrows) and transitional urinary epithelium (large arrows) are localized in close proximity. $I M$, inner medulla; $P$, papilla. Bar $=1 \mathrm{~mm}$. (C) Sense UT3 cRNA probe was hybridized to a different kidney section. A region similar to that shown in $B$ is illustrated. sistent with results from Northern analysis. Higher magnification of the papillary tip (Fig. $4 B$ ) shows that UT3 mRNA is expressed in both the papillary surface epithelium and the transitional urinary epithelium, which are in close apposition. Control experiments using sense UT3 RNA as a probe show only background hybridization (Fig. 4 C).

To determine which structures of the inner stripe of the outer medulla contain UT3, we double stained kidney sections with UT3 antisense probe and anti-von Willebrand factor antibody, a marker of the vascular endothelium (23). UT3 mRNA is found in structures which were labeled by anti-von Willebrand factor antibody, indicating that UT3 is localized in blood vessels (Fig. 5, $A$ and $B$ ). Previous studies showed that antibodies recognizing the AQP-1 water channels stain water channels in the vasa recta (26). A costaining experiment using UT3 cRNA probe and anti-AQP-1 antibody confirmed that UT3 is localized in vascular structures of the outer medulla (data not shown).

More detailed analysis of results from in situ hybridization revealed that the signals in the inner stripe of the outer medulla form the characteristic patterns of vascular bundles (Fig.
$6 \mathrm{~A}$ ). In contrast, in the inner medulla, the signals are distributed more sparsely and evenly, which is consistent with the distribution of vasa recta in this region (Fig. $6 \mathrm{~B}$ ). The signal in the papillary tip revealed that UT3 $\mathrm{mRNA}$ is present in the papillary surface epithelium and in the transitional urinary epithelium, both of which line the urinary outflow pathway (Fig. 7). Higher magnification of transitional urinary epithelium (Fig. $7 \mathrm{~B}$ ) demonstrates that the large cells at the surface of the transitional urinary epithelium are relatively poorly labeled, whereas cells in the deeper layer of the epithelium are strongly stained.

Localization of UT3 in testis. The low-magnification view in Fig. $8 A$ demonstrates that UT3 mRNA is expressed throughout the testis in a subpopulation of seminiferous tubules. Fig. $8 B$ shows that the signals reside in Sertoli cells and that some of the tubules are heavily stained, whereas others are slightly labeled or unlabeled. Thus, expression of UT3 mRNA along the seminiferous tubules is heterogeneous in a segmental fashion. To test the possibility that this heterogeneity is associated with the degree of spermatocyte maturation in individual tubules, we costained the same section with anti- 

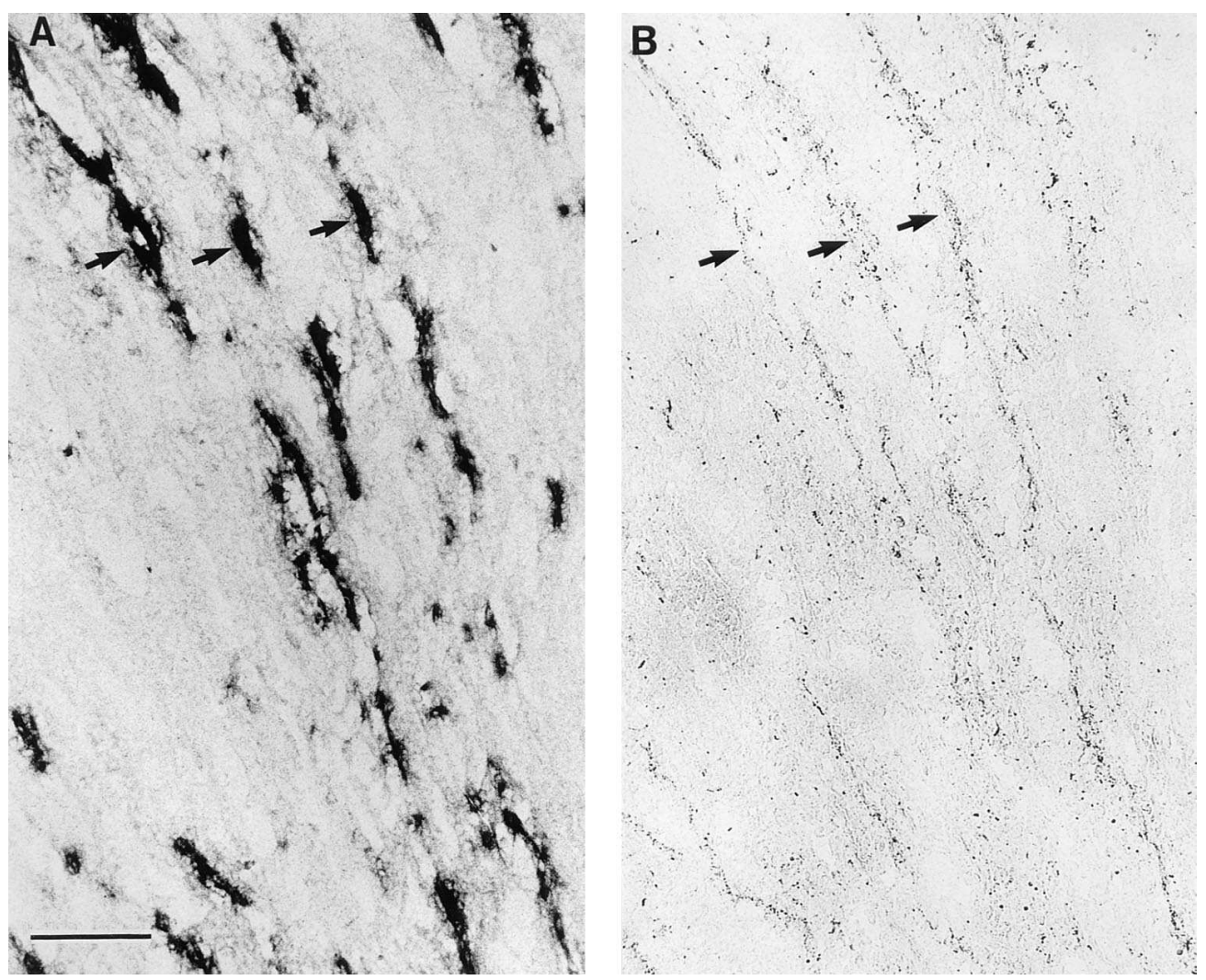

Figure 5. Colocalization of UT3 mRNA and vascular structures in the inner stripe of the outer medulla. ( $A$ and $B$ ) Adjacent sections of the inner stripe of the outer medulla were hybridized with antisense UT3 cRNA probe $(A)$ and stained with antivon Willebrand factor antibody (B). Signals of UT3 mRNA are found in the corresponding longitudinal structures similar to those in which von Willebrand factor is localized (arrows). Bar $=100 \mu \mathrm{m}$. $\alpha$-tubulin antibodies (Fig. $8 \mathrm{C}$ ). In UT3 expressing tubules, $\alpha$-tubulin staining appeared to be dot-like and scattered. In contrast, in UT3 negative tubules, $\alpha$-tubulin labeling showed the characteristic stringy appearance of more fully developed spermatozoa tails. This finding suggests that UT3 mRNA is expressed only in tubule segments containing spermatocytes in the early developmental stages.

\section{Discussion}

In the present study, we have cloned the urea transporter isoform UT3 from rat kidney inner medulla. UT3 has a high amino acid identity (80\%) to the human erythrocyte urea transporter HUT11 and is distributed widely in various organs including nonepithelial tissues such as spleen. The data indi-

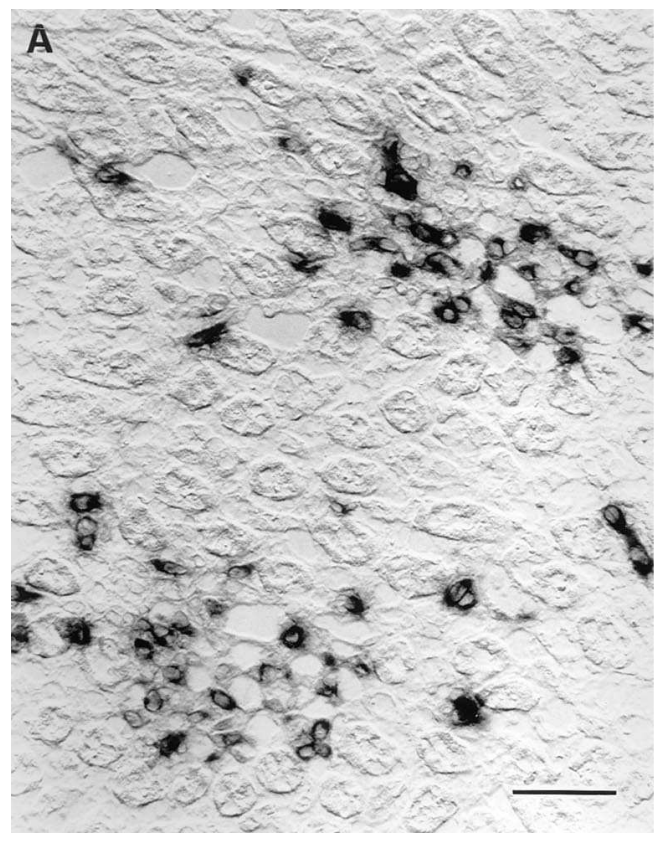

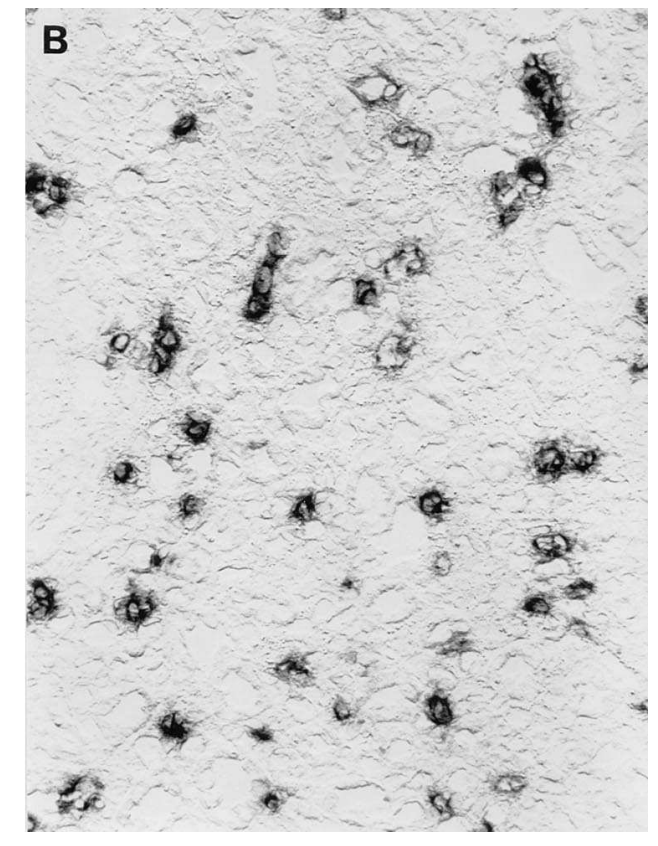

Figure 6. Localization of UT3 mRNA in the inner stripe of the outer medulla and the inner medulla. ( $A$ and $B$ ) Antisense UT3 cRNA probe was hybridized on sections of the inner stripe of the outer medulla $(A)$ and the inner medulla $(B)$. The structures containing the UT3 signals in the inner stripe of the outer medulla are arranged in small clusters, whereas positive structures in the inner medulla show a more scattered distribution.

Bar $=100 \mu \mathrm{m}$. 

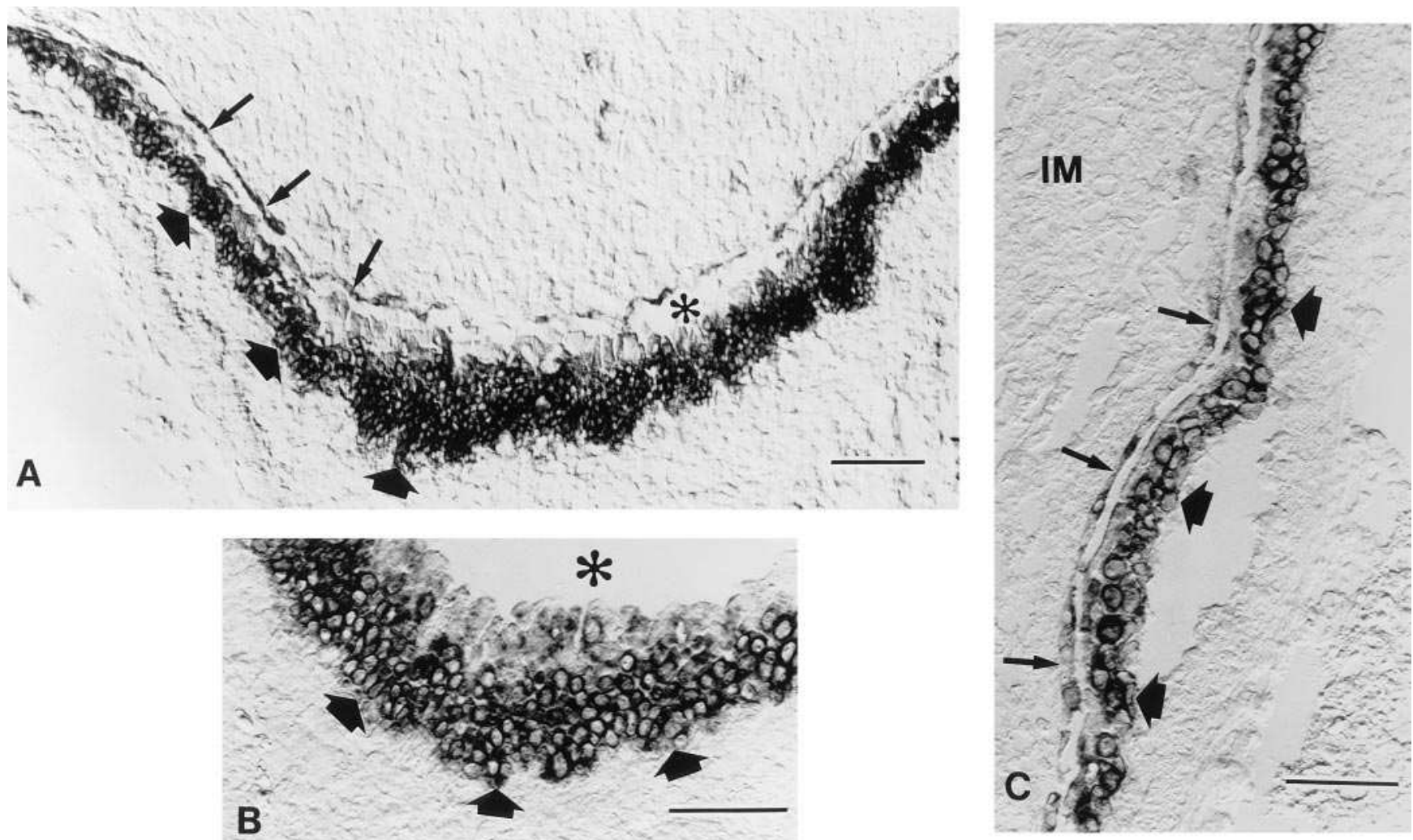

Figure 7. Localization of UT3 mRNA in the inner medulla and in the pelvic cavity. ( $A$ ) UT3 mRNA in the inner medulla. The papillary surface epithelium (small arrows) and the transitional urinary epithelium (large arrows) that surrounds the urinary space (asterisk) are both labeled. Bar $=100 \mu \mathrm{m} .(B)$ Higher magnification of UT3 staining in transitional urinary epithelium (large arrows). The large cells at the surface of the transitional urinary epithelium (top) are relatively poorly labeled, whereas cells in the deeper layer of the epithelium are strongly stained (bottom). Asterisk indicates the urinary space. Bar $=100 \mu \mathrm{m}$. (C) Higher magnification of the inner medulla (IM). The papillary surface epithelium and transitional urinary epithelium are in close apposition. Bar $=100 \mu \mathrm{m}$.

cate that UT3 is the rat homologue of HUT11 and that it corresponds to the previously reported partial clone rUT11 (12). The relevance of this study is the detailed functional characterization of rat UT3, a precise localization in kidney and testis using a double staining technique, and showing its localization in the vascular structures of the kidney, in the papillary surface epithelium, the ureter, and in the Sertoli cells of testis.

Molecular characterization and pharmacology of UT3. When expressed in Xenopus oocytes, UT3 exhibits different sensitivities to inhibitors when compared to HUT11. Comparison of the responses to the mercury compound pCMBS lead to insights into the structural-function relationships of the urea transporters UT1, UT2, UT3, and HUT11. UT3 was inhibited $32 \%$ in the presence of pCMBS $(0.5 \mathrm{mM})$, whereas HUT11 was inhibited $61 \%$ by pCMBS. In contrast, UT2 is totally resistant to pCMBS. According to the proposed membrane topology, Cys 231 is a probable target of pCMBS, because it is the only exofacial cysteine residue conserved among UT2, UT3, and HUT11. Identification of pCMBS reactive residues by a scanning mutagenesis approach would provide information regarding an urea binding site and/or urea translocation pathway.

Localization and physiological role of UT3 in kidney. The distribution of UT3 mRNA in rat kidney is different from that of UT1 and UT2. The UT3 transcript is expressed in vasa recta, most likely in DVR in the inner stripe of the outer me- dulla. It is also expressed in the surface epithelium of the papilla and the transitional epithelium of the upper part of the ureter.

The inner part of the outer medulla possesses a unique anatomical arrangement of descending limbs of short loops of Henle and vascular bundles, which consist of AVR and DVR. These structures are arranged closely together and facilitate a counter current exchange between AVR and DVR, as well as AVR and the descending limbs of short loops of Henle. AVRs arising from the inner medulla contain a relatively high urea concentration. They have a "leaky" fenestrated endothelial structure that allows exit of urea that is subsequently absorbed by DVR or short loops of Henle. It has been previously demonstrated that outer medullary DVR represent an essential pathway for countercurrent exchange of urea (1-3). In vitro microperfusion showed that DVR contain a urea transporter that is inhibited $40,20-30$, and $50 \%$ by $200 \mathrm{mM}$ thiourea, 0.5 $\mathrm{mM}$ phloretin, and $0.5 \mathrm{mM}$ pCMBS, respectively (15). This inhibition profile is similar to UT3 expressed Xenopus oocytes. Thus, it is reasonable to assume that urea exits AVR via its fenestrated structures and enters DVR via UT3, thereby minimizing urea escape from the inner medulla to systemic circulation.

In situ hybridization (Fig. 6) showed that UT3 signals are found not only in the outer medullary DVR but also in the papillary DVR. This finding is against the results of in vivo mi- 

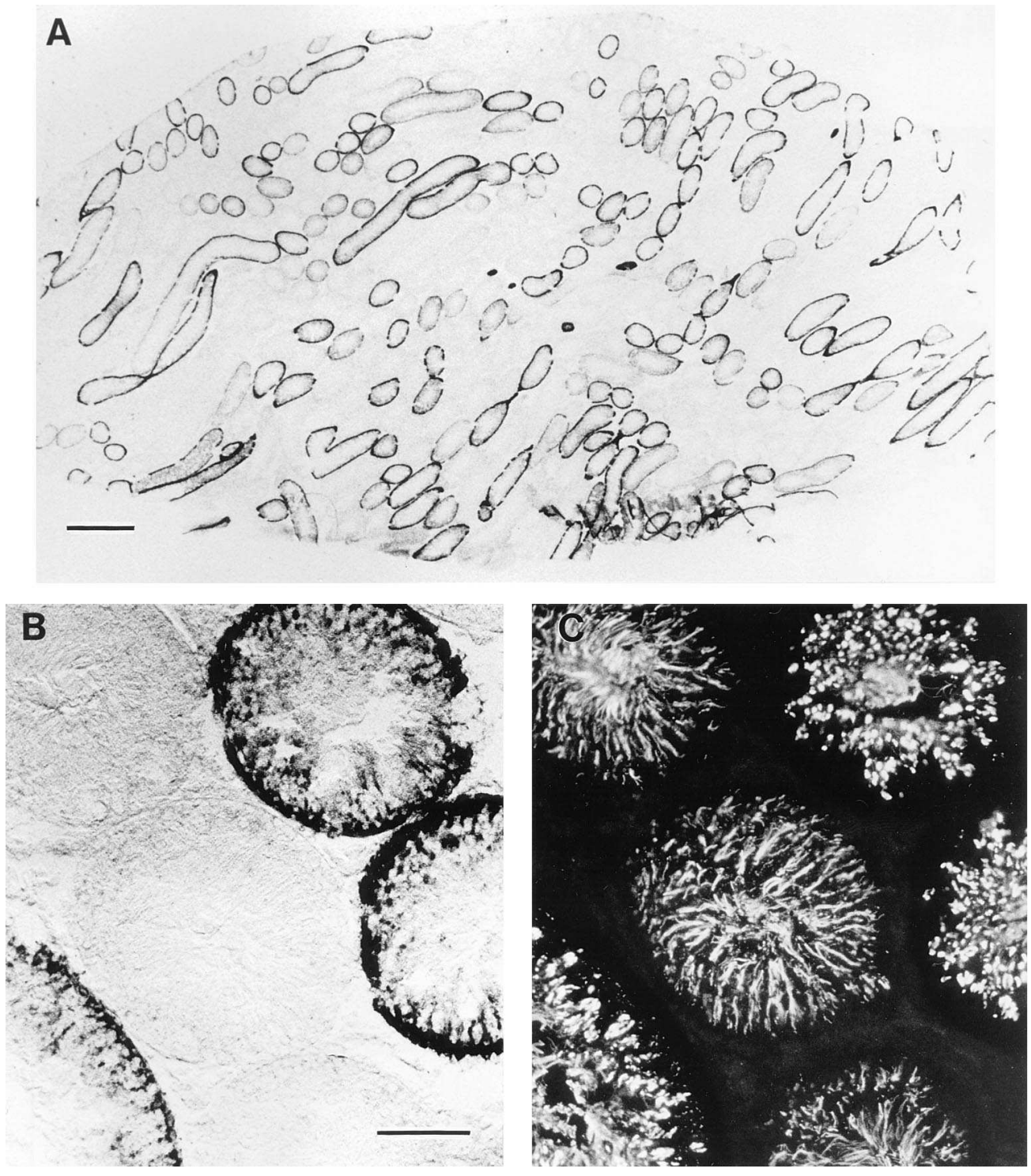

Figure 8. Localization of UT3 mRNA in testis. (A) UT3 cRNA probe was hybridized on a section of rat testis. UT3 is expressed throughout the testis in a subpopulation of seminiferous tubules. Bar $=1 \mathrm{~mm}$. $(B)$ Higher magnification of cross section of seminiferous tubules. The UT3 signals are detected in Sertoli cells, which line the perimeter of the tubules. Bar = $100 \mu \mathrm{m}$. (C) Staining of the same section with anti- $\alpha$-tubulin antibody. In UT3 positive tubule segments, the signals appear to be punctate and scattered. In contrast, in UT3 negative segments, staining displays a characteristic rope-like appearance of mature spermatozoa tails. Bar $=100 \mu \mathrm{m}$.

croperfusion study (27), which suggests the absence of urea transporters in the papillary DVR. It is possible that the discrepancy is due to an underestimation of urea permeability in the microperfusion studies and/or to a strain-difference of UT3 expression between Munich-Wistar rats (microperfusion study) and Sprague-Dawley rats (in situ hybridization).

The presence of UT3 in the surface epithelium of the renal papilla and pelvis is in agreement with the hypothesis that the renal pelvis contributes to urea accumulation in the inner medulla. Superfusion studies showed that urea can penetrate the papillary surface epithelium of the renal pelvis, and thereby reenter the medullary interstitium (28). On the other hand, another study suggested that the renal pelvis is not a major source of urea recycling, because the permeability and surface area of the papillary surface epithelium are too small to exert a physiological effect (29). Analysis of its physiological significance awaits further experiments addressing the contribution of urea movement from the papillary surface epithelium to the maintenance of the cortico-papillary osmolarity gradient. With regard to the ureter, a micropuncture study (30) showed that $\sim 7 \%$ of the urea from the pelvic outflow is absorbed between the proximal and distal parts of the ureter before reaching the bladder. Thus, UT3 mRNA expression in the ureter might be associated with the urea absorption in this region.

Physiological role of UT3 in testis. Our finding that UT3 mRNA is expressed in Sertoli cells sheds light on a novel physiological aspect of urea transporters. Micropuncture studies using rat testis revealed that urea can be transported from blood to the lumen of seminiferous tubules (31). However, little is known about the significance of urea transport in Sertoli cells. The main function of Sertoli cells is thought to be to support and control germ cell differentiation by providing nutrients and/or regulatory factors (32). Interestingly, UT3 expression is heterogeneous in cross sections of seminiferous tubules. Double staining experiments with anti- $\alpha$-tubulin antibody showed that the tubule sections expressing UT3 mRNA contain immature spermatocytes, which have not formed long tails. Since germ-cell differentiation in the seminiferous epithelium progresses in a segmental fashion (32), our results suggest that UT3 is upregulated in the early stages of spermato- 
cyte development. A possible role of UT3 in Sertoli cells is that it allows exit of urea which is formed during the synthesis of arginine and ornithine, both of which are intermediates of the urea cycle $(1,3)$. Arginine is an essential component of protamine, a sperm-specific protein (33). Ornithine is an obligatory precursor of the synthesis of polyamines, such as spermidine and spermine (34). Thus, Sertoli cells may need to excrete large amounts of urea arising from the polyamine pathway and, therefore, express more urea transporters. Because polyamine synthesis in Sertoli cells is known to be induced rapidly in response to accelerated cell-division of germ cells (34), it is reasonable to predict that there is a regulatory mechanism to promote urea transport expression. Understanding the role of urea transport in spermatogenesis may lead to insights relevant to the pathogenesis of infertility.

Expression of UT3 in other tissues. A striking feature of the distribution of UT3 is its widespread expression in brain, which has an mRNA abundance similar to kidney. Little is known about the significance of urea transport in the brain. Previous studies demonstrated that brain contains urea at levels similar to those in liver (35). Although the brain per se can produce urea, the brain, unlike liver, lacks carbamyl phosphatase and ornithine transcarbamylase, which are both essential for citrulline synthesis (36). Thus, this partial defect of urea cycle enzymes suggests that urea formation in brain has no significant involvement in the detoxification of ammonia. Further localization and physiological studies are required to elucidate the role of UT3 in brain.

The moderate degrees of expression of UT3 in spleen, bone marrow, and thymus can be explained by the fact that blood cells expressing UT3 are pooled in these organs. Low levels of UT3 expression in lung could account for the weak signal ( $\sim 4 \mathrm{~kb}$ ) for lung on Northern analysis of rabbit UT2 under low stringency conditions (6).

It should be noted that no UT3 transcripts have been observed in the liver. The presence of a liver urea transporter has been postulated, since liver is a major site of urea production and therefore needs to excrete urea into blood (1-3). Previous studies using rat liver perfusion (37) and liver mRNA expression in Xenopus oocytes (38) suggested that there is a cAMPindependent and phloretin-inhibitable urea transporter in the liver. Given the lack of UT1, UT2, and UT3 hybridization signals in rat liver (7-9), this suggests that the liver urea transporter is structurally different from the known urea transporter family members.

\section{Acknowledgments}

This work was supported by the National Institutes of Health grants R01-DK 707844 to M.A. Hediger, R01-DK 42956 to D. Brown, and the research fellowship from National Kidney Foundation to $\mathrm{H}$. Tsukaguchi, and from the Siriraj-China Medical Board, Mahidol University, Thailand to C. Shayakul.

\section{References}

1. March, D.J., and M.A. Knepper. 1992. Renal handling of urea. In Renal Physiology. E.E. Windhager, editor. Oxford University Press, Oxford, United Kingdom. 1317-1347.

2. Knepper, M.A., and F. Roch-Ramel. 1987. Pathways of urea transport in the mammalian kidney. Kidney Int. 31:629-633.

3. Bankir, L. 1995. Urea and the kidney. In The Kidney. 5th ed. B.M. Brenner and F.C. Rector, Jr., editors. W.B. Saunders Company, Philadelphia. 571-
606

4. Chou, C.L., and M.A. Knepper. 1990. Concentration dependence of urea and thiourea transporter in rat inner medullary collecting. Am. J. Physiol. 258: F486-F494.

5. Macey, R.I., and Y.W. Yousef. 1988. Osmotic stability of red cells in renal circulation requires rapid urea transport. Am. J. Physiol. 254:C669-C674.

6. You, G., C.P. Smith, Y. Kanai, W.-S. Lee, M. Stelzner, and M.A. Hediger. 1993. Cloning and characterization of the vasopressin-regulated urea transporter. Nature (Lond.). 365:844-847.

7. Smith, C.P., W.-S. Lee, S. Martial, G. You, J.M. Sands, and M.A. Hediger. 1995. Cloning and regulation of expression of the rat kidney urea transporter (rUT2). J. Clin. Invest. 92:2448-2457.

8. Hediger, M.A., C.P. Smith, G. You, W.-S. Lee, Y. Kanai, and C. Shayakul. 1996. Structure, regulation, and physiological roles of urea transporters. Kidney Int. 49:1615-1623.

9. Shayakul, C., A. Steel, and M.A. Hediger. 1996 Molecular cloning and characterization of the vasopressin-regulated urea transporter of rat kidney collecting ducts. J. Clin. Invest. 98:2580-2587.

10. Olives, B., P. Neau, P. Bailly, M.A. Hediger, G. Rousselet, J.P. Cartron, and P. Ripoche. 1994. Cloning and functional expression of a urea transporter from human bone marrow cells. J. Biol. Chem. 269:31649-31652.

11. Olives, B., M.G. Mattei, M. Huet, P. Neau, S. Martial, J.P. Cartron, and P. Bailly. 1995. Kidd blood group and urea transport function of human erythrocyte are carried by the same protein. J. Biol. Chem. 270:15607-15610.

12. Promeneur, D., G. Rousselet, L. Bankir, P. Bailly, J.P. Cartron, P. Ripoche, and M-M. Trinh-Trang-Tan. 1996. Evidence for distinct vascular and tubular urea transporters in the rat kidney. J. Am. Soc. Nephrol. 7:852-860.

13. Knepper, M.A., and R.A. Star. 1990. The vasopressin-regulated urea transporter in renal inner medullary collecting duct. Am. J. Physiol. 259:F393F401.

14. Sands, J.M., and J.P. Kokko. 1987. Countercurrent exchange. Kidney Int. 38:695-699.

15. Pallone, T.L. 1994. Characterization of the urea transporter in outer medullary descending vasa recta. Am. J. Physiol. 267:R260-R267.

16. Pallone, T.L., J. Work, R.L. Myers, and R.L. Jamison. 1994. Transport of sodium and urea in outer medullary descending vasa recta. J. Clin. Invest. 93: 212-222.

17. Shayakul, C., M.A. Knepper, C.P. Smith, S.R. DiGiovanni, and M.A. Hediger. 1996. FASEB J. 10:273a. (Abstr.)

18. Nielsen, S., J. Terris, C.P. Smith, M.A. Hediger, C.A. Ecelbarger, and M.A. Knepper. 1996. Cellular and subcellular localization of the vasopressinregulated urea transporter in rat kidney. Proc. Natl. Acad. Sci. USA. 93:54955500 .

19. Imai, M., M. Hayashi, and M. Araki. 1984. Functional heterogeneity of the descending limbs of Henle loop. I. Internephron heterogeneity of the hamster kidney. Plugers Arch. 402:393-401.

20. Fushimi, K., S. Uchida, Y. Hara, Y. Hirata, F. Marumo, and S. Sasaki. 1993. Cloning and expression of apical membrane water channel of rat kidney collecting tubule. Nature (Lond.). 361:549-552.

21. Agre, P., D. Brown, and S. Nielsen. 1995. Aquaporin water channel: unanswered questions and unresolved controversies. Curr. Opin. Cell Biol. 7:472483.

22. Schaeren-Wiemers, N., and A. Gerfin-Moser. 1993. A single protocol to detect transcripts of various types and expression levels in neural tissue and cultured cells: in situ hybridization using digoxigenin-labeled cRNA probes. Histochemistry. 100:431-440.

23. Nadji, M., M.S. Gonzales, A. Castro, and A.R. Morales. 1985. Factor VIII-related antigen: an endothelial cell marker. Lab. Invest. 42:139.

24. Piperno, G., M. LeDizet, and X.-J. Chang. 1987. Microtubules containing acetylated $\alpha$-tubulin in mammalian cells in culture. J. Cell Biol. 104:289-302.

25. Kozak, M. 1991. Structural features in eukaryotic mRNA that modulate the initiation of translation. J. Biol. Chem. 266:19867-19870.

26. Sabolic, I., G. Valenti, J.M. Verbavatz, A.N. Van Hoek, A.S. Verkman, D.A. Ausiello, and D. Brown. 1992. Localization of the CHIP28 water channel in rat kidney. Am. J. Physiol. 263:C1225-C1233.

27. Pallone, T.L., S. Nielsen, E.P. Silldorff, and S. Yang. 1995. Diffusive transport of solute in the rat medullary microcirculation. Am. J. Physiol. 269: F55-F63.

28. Bonventre, J.V., R.J. Roman, and C. Lechene. 1980. Effect of urea concentration of pelvic fluid on renal concentrating ability. Am. J. Physiol. 239: F609-F610.

29. Sands, J.M., and M.A. Knepper. 1987. Urea permeability of mammalian inner medullary collecting duct system and papillary surface epithelium. J. Clin. Invest. 79:138-147.

30. Walser, B.L., Y. Yagil, and R.L. Jamison. 1988. Urea flux in the ureter. Am. J. Physiol. 255:F244-F249.

31. Howards, S.S., and T.T. Turner. 1979. The blood testis barrier: a mor phologic or physiologic phenomenon. Trans. Am. Assoc. Genito. Surgeons. 70: 74-75.

32. Weiss, L. 1988. The male reproductive systems. In Cell and Tissue Biology, 6th edition, Urban and Schwarzenberg, Baltimore. 947-953.

33. Krawetz, S.A., and G.H. Dixon. 1988. Sequence similarities of the prot- 
amine genes: implications for regulation and evolution. J. Mol. Evol. 27:291297.

34. Tabor, C.W., and H. Tabor. 1984. Polyamines. Annu. Rev. Biochem. 53: 749-790

35. Buniatian, C.H., and M.A. Davtian. 1962. Urea synthesis in brain. $J$. Neurochem. 13:743-753.

36. Arnt-Ramos, L.R., W.E. O'Brien, and S.R. Vincent. 1992. Immunocytochemical localization of argininosuccinate synthetase in the rat brain to nitric oxide synthesis-containing neurons. Neuroscience. 51:773-789.

37. Effros, R., E. Jacobs, A. Hacker, K. Ozker, and C. Murphy. 1993. Reversible inhibition of urea exchange in rat hepatocytes. J. Clin. Invest. 91:28222828 .

38. Hasegawa, H., and A.S. Verkman. 1993. Functional expression of cAMP dependent and independent urea transporters in Xenopus oocytes. Am. J. Physiol. 265:C514-C520. 